Communication

\title{
Laterality of the activation of the vastus lateralis muscle in females with Parkinson's disease during the medication state compared with healthy controls
}

\author{
Yuichi Nishikawa ${ }^{1, *}$, Kohei Watanabe ${ }^{2}$, Tetsuya Takahashi ${ }^{3}$, Noriaki Maeda ${ }^{4}$, Hirofumi Maruyama ${ }^{5}$, Shinobu Tanaka ${ }^{1}$, \\ Allison Hyngstrom ${ }^{6}$ \\ ${ }^{1}$ Faculty of Frontier Engineering, Institute of Science \& Engineering, Kanazawa University, 9201192 Kanazawa, Japan \\ ${ }^{2}$ Laboratory of Neuromuscular Biomechanics, School of Health and Sport Sciences, Chukyo University, 4700393 Nagoya, Japan \\ ${ }^{3}$ Depeatment of Rehabilitation, Faculty of Rehabilitation, Hiroshima International University, 7392695 Hiroshima, Japan \\ ${ }^{4}$ Division of Sports Rehabilitation, Graduate School of Biomechanical and Health Sciences, Hiroshima University, 7348551 Hiroshima, Japan \\ ${ }^{5}$ Department of Clinical Neuroscience and Therapeutics, Hiroshima University, 7348551 Hiroshima, Japan \\ ${ }^{6}$ Department of Physical Therapy, Marquette University, Milwaukee, WI 53201, USA \\ *Correspondence: yuichi@se.kanazawa-u.ac.jp (Yuichi Nishikawa)
}

DOI:10.31083/j.jin2101040

This is an open access article under the CC BY 4.0 license (https://creativecommons.org/licenses/by/4.0/).

Submitted: 21 April 2021 Revised: 21 May 2021 Accepted: 22 July 2021 Published: 28 January 2022

This study quantified the laterality of motor unit activation properties in females with Parkinson's disease during force production (low to high-intensity contraction) using high-density surface electromyography. Sixteen females with Parkinson's disease (age $=69.9 \pm 7.6$ years, disease duration $=4.9 \pm 5.1$ years) and 14 healthy female subjects (age $=68.6 \pm 3.6$ years) performed submaximal ramp-up contractions during isometric knee extension. High-density surface electromyography signals were recorded from both vastus lateralis muscles. The level of heterogeneity was calculated in the spatial distribution patterns of the high-density surface electromyography signals to determine the modified entropy, coefficient of variation of the root mean square and correlation coefficient to evaluate motor unit activation properties. Pearson's correlation coefficients were calculated to examine the relationships between disease severity and the root mean square and electromyography variables. The root mean square value and heterogeneity were significantly higher and lower on the more-affected side in females with Parkinson's disease than on the contralateral side in females with Parkinson's disease or either side in control subjects $(p<0.05)$. Females with Parkinson's disease that exhibited temporal changes of spatial motor unit activation properties showed significant laterality when compared to healthy control subjects in both the low and high-intensity contractions. Moderate-tostrong correlations were observed between disease severity and root mean square and electromyography variables in females with Parkinson's disease $(r>0.6, p<0.001)$. The laterality of motor unit activation properties was compared between the subjects with Parkinson's disease and the control subjects. The findings suggest that females with Parkinson's disease have asymmetrical motor unit activation properties, independent of the magnitude of force production.

\section{Keywords}

Motor unit; Vastus lateralis muscle; Parkinson's disease; Isometric contraction; Laterality

\section{Introduction}

Parkinson's disease (PD) is a progressive neurodegenerative disorder characterized by unilateral motor symptoms [1]. These symptoms are caused by asymmetric central nervous system degeneration [2]. The central nervous system regulates motor unit (MU) recruitment and firing rates that regulate force output [3]. Previous studies using intramuscular electromyography (EMG) and high-density surface EMG (HD-SEMG) have shown that people with PD exhibit higher MU firing rates than healthy subjects $[4,5]$. In particular, they have reported that people with PD have higher amplitudes of MU activity during low-intensity muscle contraction [5, 6]. Although MUs are newly recruited as the force increases in accordance with the size principle [7], Nishikawa et al. [5] reported that the more-affected side of females with PD deviates from the muscle activity pattern according to the size principle and shows abnormal MU firing behavior. Furthermore, Glendinning et al. [4] reported a possible shift in the MU population to lower recruitment thresholds in people with PD compared with healthy subjects.

Clinically, people with PD often present with asymmetry of physical symptoms (such as tremor and rigidity) [8] and MU firing behavior has been reported to differ between the more- and less-affected sides [5]. However, those studies analyzed MU firing behavior only during low-intensity muscle contraction. It is not clear what activity patterns MUs exhibit during high-intensity muscle contraction. Another study reported that people with PD experience selective atrophy of fast-twitch muscle fibers in addition to degeneration of the central nervous system [9]. Therefore, there may be a limitation of MU activation properties during high-intensity force production in people with PD. Although needle EMG 
Table 1. Characteristics of the participants.

\begin{tabular}{lcc}
\hline Variables (unit) & Parkinson's disease & Control subjects \\
\hline Age (years) & $69.9 \pm 7.6$ & $68.6 \pm 3.6$ \\
Height $(\mathrm{cm})$ & $155.9 \pm 9.4$ & $153.3 \pm 3.6$ \\
Weight $(\mathrm{kg})$ & $54.1 \pm 8.9$ & $54.9 \pm 6.5$ \\
Knee extension torque (Nm) & $53.0 \pm 10.0^{*} / 69.5 \pm 21.0$ & $67.3 \pm 12.8 / 66.5 \pm 12.6$ \\
More-affected side/Less-affected side or Right/Left & $4.9 \pm 5.1$ & N/A \\
Disease duration (years) & $9(1-14)$ & \\
UPDRS part III &
\end{tabular}

Data are shown as mean \pm SD or median (min-max).

* Significant difference between contralateral side of people with PD and control subjects.

is used to evaluate MU activation properties, it is painful and unsuitable for measuring the activity of MUs during highintensity muscle contraction while HD-SEMG has the advantage of collecting similar information both noninvasively and painlessly.

HD-SEMG is a recently developed noninvasive method for evaluating individual MU activation properties using multiple electrodes (e.g., 64-400 channels) and spatial activation patterns across an entire muscle (Fig. 1A) [10,11]. Studies that use this technique have shown that the spatial distribution of HD-SEMG activation across a muscle is affected by the intensity of contractions or fatigue level [12-14]. This phenomenon has been explained by the presence of spatial heterogeneity in the locations of different types of muscle fibers [15] and the clustering of muscle fibers innervated by one MU in a defined area [16]. Accordingly. changes in the spatial distribution of HD-SEMG signals can be explained by differences in MU recruitment patterns across the muscle, suggesting that the spatial distribution of HD-SEMG signals can be used to study changes in MU recruitment $[17,18]$. Another study documented higher overall amplitudes of SEMG signals and lower heterogeneity in the SEMG signal distribution on the more-affected side of people with PD during force production than in healthy subjects [6]. The lower level of heterogeneity in the spatial EMG potential distribution may be due to the smaller number of newly recruited MUs in people with PD. However, to the best of the author's knowledge, no studies have identified the laterality of MU activation properties in people with PD during high-intensity muscle contraction or the relationship with clinical measures of function.

The purpose of this study was to examine the laterality of the spatial distribution pattern of MU activation properties during force production (low- to high-intensity contraction) using HD-SEMG. It was hypothesized that compared with controls, people with PD would exhibit laterality of MU activation properties.

\section{Materials and methods}

\subsection{Subjects}

Sixteen idiopathic females with PD and 14 age-matched female controls were enrolled in the present study (Table 1).
A previous study reported that females with PD exhibited larger asymmetries of motor symptoms than males with PD [19]. Thus, gender is an important factor in the unilateral symptoms of PD. For this reason only females with PD were recruited for this study. The exclusion criteria included: Hoehn \& Yahr stage $>3$, diagnosis of orthopedic disorders (e.g., knee and hip osteoarthritis, anterior cruciate ligament injury) and other neurological diseases (e.g., Alzheimer's disease, amyotrophic lateral sclerosis, dystonia and peripheral nerve disorders). All experimental procedures were performed in accordance with the Declaration of Helsinki. The research protocol was approved by the Hiroshima University Committee on Ethics in Research (approval number, E53-2). All participants received verbal and written explanations about their participation in the study and publication of this article and signed a consent form. Assessment of physical function and EMG were performed for all participants in the on-medication state.

\subsection{Experimental protocols}

All participants underwent measurement of maximal voluntary isometric contraction (MVIC) of the bilateral knee extensors. The order of the measurements was randomized to avoid bias toward the more or the less-affected side (or toward the right or left side). A Dynamometer (Biodex System 4; Biodex Medical Systems, Shirley, NY, USA) was used to measure the MVIC. During measurement, the hip and knee joint angles were fixed at $90^{\circ}$ (Fig. 1B) [20]. All participants were measured in two MVIC trials, with a break of at least two minutes between trials and a warm-up period of ten minutes before assessment [20]. The target torque for the submaximal ramp-up contractions was calculated from the peak MVIC torque. After MVIC assessments were recorded, all participants performed a submaximal ramp-up isometric contraction from 0 to $70 \%$ of the MVIC (the ramp-up rate was approximately $10 \%$ of MVIC/sec; Fig. 1C) $[6,21]$. The target torque and torque generated by a participant were displayed on a monitor. Physical function in people with PD was assessed by the Unified Parkinson's Disease Rating Scale (UPDRS) and was performed by the same neurologist within a week before and after EMG measurement. Both the more and less-affected side in people with PD were defined by UPDRS score and their anamnesis. 

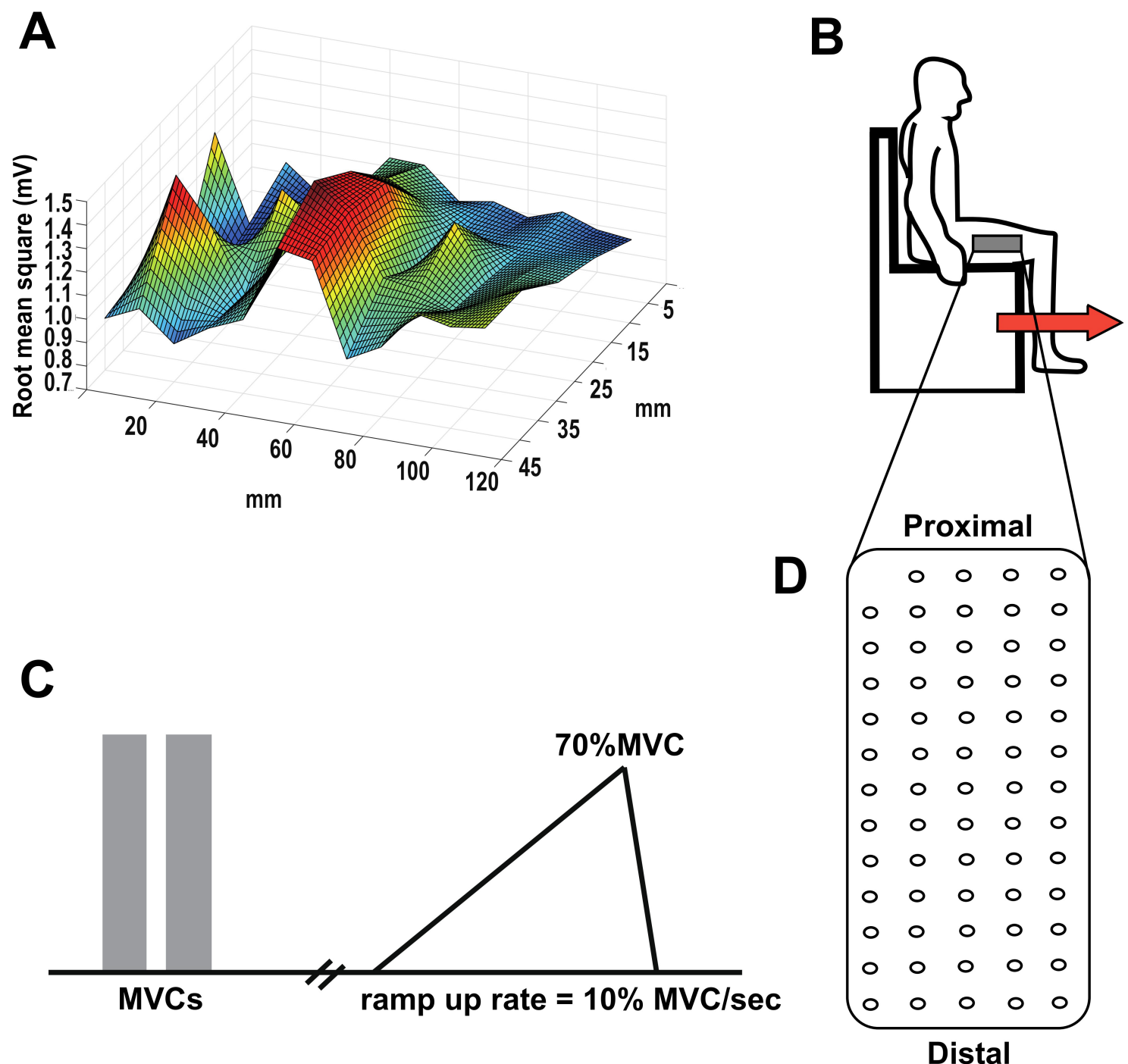

Fig. 1. High-density SEMG evaluates the spatial distribution pattern of muscle activity within a muscle. (A) Topographic map of the SEMG RMS values at $10 \%$ MVIC of a control subject (age 67). (B) During measurement, the hip and knee joint angles were fixed at $90^{\circ}$. (C) Experimental protocol. After MVIC measurements were recorded, all subjects performed ramp-up contractions. (D) The two-dimensional electrode grid consisted of 64 electrodes (interelectrode distance; $8 \mathrm{~mm}$ in each direction).

\subsection{EMG recording}

HD-SEMG signals were detected from both sides of the VL muscle using a two-dimensional grid of 64 electrodes (ELSCH064NM2; OT Bioelettronica, Torino, Italy) during the MVIC and submaximal ramp-up contraction in accordance with previous studies $[6,20,22,23]$. The multielectrode grid consisted of thirteen rows and five columns (interelectrode distance, $8 \mathrm{~mm}$ in each direction, Fig. 1D). The tested area was shaved and cleaned with alcohol. Multiple electrodes were attached to the skin using a biadhesive sheet (KITAD064NM2; OT Bioelettronica) after adaptation of the conductive paste (Elefix Z-181BE; Nihon Kohden, Tokyo, Japan). A multiple electrode was attached at the center of the line between the femoral greater trochanter and the lateral edge of the patella. A ground electrode was placed at the anterior superior iliac spine [6, 20, 22, 23]. All EMG measurements were performed by the same examiner.

The monopolar signals were amplified (off-line bandpass filtered $10-500 \mathrm{~Hz}$ ) by a factor of 1000 , sampled at $2048 \mathrm{~Hz}$, digitized by a 12-bit A/D converter (EMG-USB2+, OTBioelettronica) and processed using MATLAB software (MATLAB 2019b, Math Works GK, MA, USA). The root mean square (RMS) of EMG signals was calculated for a section of $500 \mathrm{~ms}$ centered at each 10\% increase in MVIC force up to $70 \%$ MVIC during the submaximal ramp-up contraction task [6]. The RMS values of each electrode during the submaximal ramp-up contraction task were normalized by the RMS values of the MVIC.

The modified entropy and coefficient of variation $(\mathrm{CoV})$ of the RMS were used to analyze the measurement of the 
heterogeneity of the spatial distribution pattern of the HDSEMG amplitudes in each section. The modified entropy was calculated from the RMS values of each specific torque during submaximal ramp-up contraction (i.e., 10\% to 70\% MVIC) in accordance with a previous study [24]. The RMS coefficient of variation $(\mathrm{CoV})$ was calculated from the mean and standard deviation of the 59 RMS measurements at each specific torque level (i.e., 10\% to 70\% MVIC) [6]. The changes in these variables indicate changes in the spatial distribution pattern of the MU activation properties within a muscle. A lower modified entropy and a higher CoV of the RMS indicate more heterogeneity in the spatial EMG distribution pattern within the multiple electrodes $[25,26]$. Correlation coefficients were calculated from the RMS value of each channel between 10\% MVIC and the other torque levels (i.e., 20\% to $70 \%$ MVIC) to compare the temporal changes in the spatial distribution pattern of MU activation. The modified entropy and $\mathrm{CoV}$ of the RMS provide insight into the regulation of the nervous system for muscle activation patterns within a muscle, irrespective of the magnitude of activation [27]. The correlation coefficients provide information about temporal changes in the activation properties of MUs [21].

\subsection{Statistical analyses}

TResults are presented as the mean \pm SD or medians (min-max). Before the statistical analyses, data normality was assessed with the Shapiro-Wilk test. The Kruskal-Wallis test and Dunn's test for multiple comparisons were used to compare knee extension torque. The normalized EMS, modified entropy and CoV of the RMS were analyzed using a mixed-effects model (explanatory variables: group-PD or control, limb-more-affected side or less-affected side, right or left side and their interaction terms; continuous variable: \% MVIC). Correlation between scores of UPDRS part III and $\Delta$ normalized RMS, $\Delta$ modified entropy and $\Delta \mathrm{CoV}$ of the RMS were assessed using Spearman's correlation coefficient. Two-way analysis of variance (factors: group [PD or control] and torque [20\%, 30\%, 40\%, 50\%, 60\%, and 70\% MVIC]) and multiple pairwise $t$-tests with the Bonferroni correction were used to compare values of the $\Delta$ correlation coefficient. The $\Delta$ EMG variables ( $\Delta$ normalized RMS, $\Delta$ modified entropy, $\Delta \mathrm{CoV}$ of the RMS and $\Delta$ correlation coefficient) were calculated as the difference between two sides in people with PD. The following thresholds of correlation coefficient were used to determine the correlation strength: $0.2 \leq r \leq 0.4$ weak correlation, $0.4 \leq r \leq 0.7$ moderate correlation, 0.7 $\leq r \leq 0.9$ strong correlation, and $0.9 \leq r \leq 1.0$ very strong correlation [28]. Statistical analyses were performed using SPSS (version 25, SPSS, Inc., Chicago, IL, USA). The level of significance was chosen as $p<0.05$.

\section{Results}

The knee extension torque on the more-affected side of people with PD was significantly smaller than on the contralateral sides of people with PD and control subjects $(p<$ 0.05, Table 1).
A

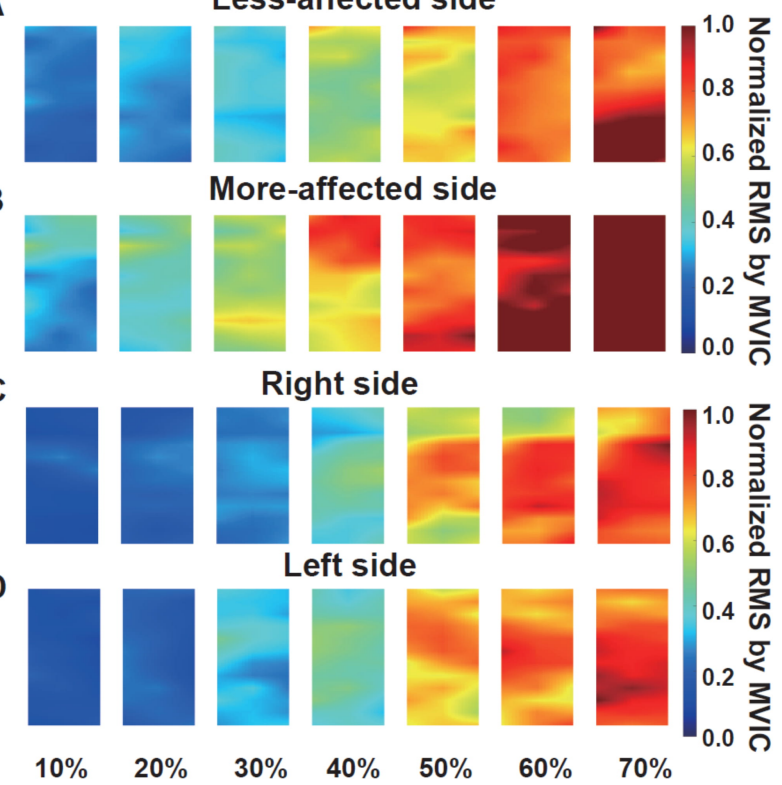

Knee extension torque (\%MVIC)

Fig. 2. Topographic map of HD-SEMG amplitudes during the submaximal ramp-up contraction task. The RMS was normalized to the maximal voluntary contraction value.(A) The less-affected side in PD subjects. (B) The more-affected side in PD subjects. (C) The right side of control subjects. (D) The left side of control subjects.

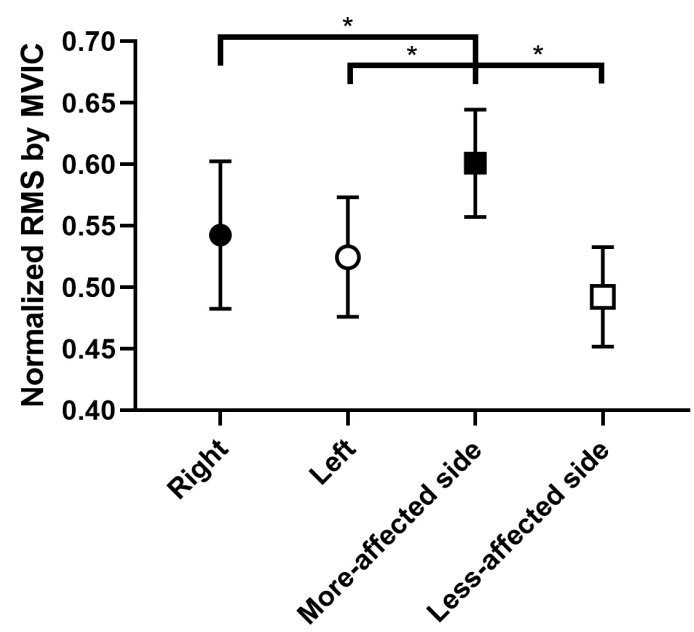

Fig. 3. Comparison of the normalized RMS between the two sides of control subjects and PD subjects. The more-affected side in PD subjects showed a significantly higher normalized RMS value than the contralateral side in PD subjects or either side in control subjects. ${ }^{*} p<0.05$. Data are presented as mean $\pm 95 \%$ CI.

A topographic map of HD-SEMG amplitude on both sides of people with PD and control subjects is shown in Fig. 2AD. Marked differences were evident in the spatial distribution of the HD-SEMG signals at each torque level. In this example and across the dataset in general, the normalized RMS magnitude of the more-affected side of a person with PD was 
A

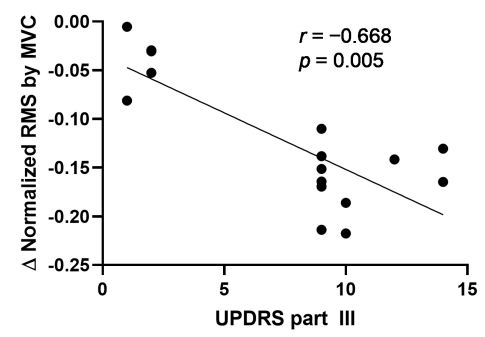

B

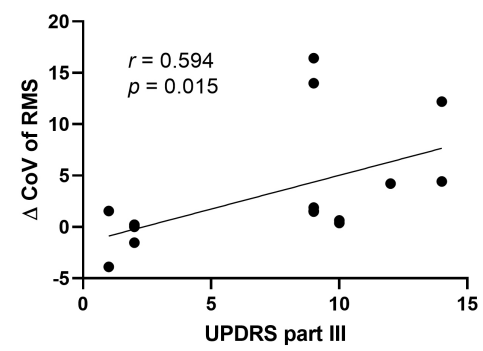

C

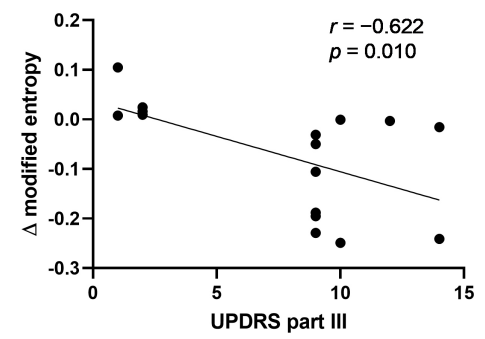

Fig. 4. Association between the UPDRS part III and EMG variables during the submaximal ramp-up contraction task. Correlations between UPDRS part III scores and $\Delta$ normalized RMS values (A), CoV of RMS (B), and $\Delta$ modified entropy (C). Moderate correlations were observed between the UPDRS part III scores and the above three variables, indicating that subjects with higher severity of physical symptoms had more unilateral motor unit activation properties.
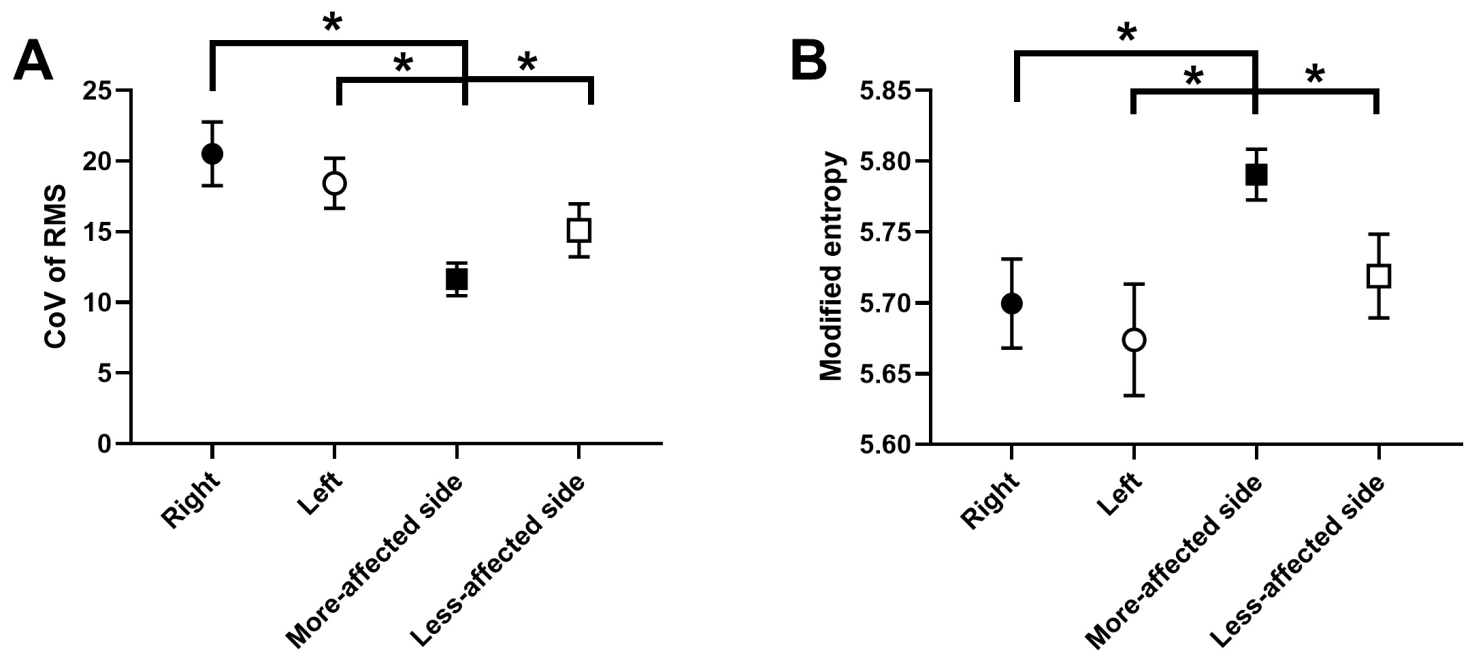

Fig. 5. Comparison of EMG variables during the submaximal ramp-up contraction task. The CoV of the RMS (A) and modified entropy (B) between the two sides of control subjects and people with PD. The more-affected side in the people with PD showed a significantly lower CoV of the RMS and a significantly higher modified entropy than the contralateral side in people with PD or either side in control subjects. ${ }^{*} p<0.05$. Data are presented as mean $\pm 95 \%$ CI.

larger than the contralateral side of people with PD and control subjects. The normalized RMS significantly differed between the two groups $(p<0.001)$ and the more-affected side of people with $\mathrm{PD}$ showed significantly higher values than the contralateral side of people with PD and control subjects $(p<0.05$, Fig. 3).

Moderate correlations were observed between the scores of UPDRS part III and $\Delta$ normalized RMS values, $\Delta \mathrm{CoV}$ the RMS and $\Delta$ modified entropy during submaximal ramp-up contractions $(r=-0.688, p=0.005 ; r=0.594, p=0.015 ; r=$ $-0.622, p=0.010$, respectively, Fig. 4A-C).

The CoV of the RMS and modified entropy differed significantly between the groups $(p<0.001)$ and the moreaffected side of the PD subjects showed significantly lower and higher values, respectively, than the contralateral side of PD subjects and control subjects $(p<0.001$, for each, Fig. 5AB).
The $\Delta$ correlation coefficient did not show a significant interaction between torque and group $(p=0.363)$. The results of two-way ANOVA showed that $\Delta$ correlation coefficient was significantly higher in the people with $\mathrm{PD}$ than in the control subjects ( $p=0.002$, Fig. 6).

\section{Discussion}

The present study compared the laterality of MU activation properties between people with PD and control subjects. The primary results were that the more-affected side in people with PD exhibited (1) higher normalized RMS values and (2) less heterogeneity than the contralateral side in people with PD or either side in control subjects. (3) Furthermore, people with PD exhibited moderate correlations between UPDRS part III scores and the laterality of EMG variables ( $\Delta$ normalized RMS, $\Delta \mathrm{CoV}$ of RMS and $\Delta$ modified entropy) during submaximal ramp-up contraction and 


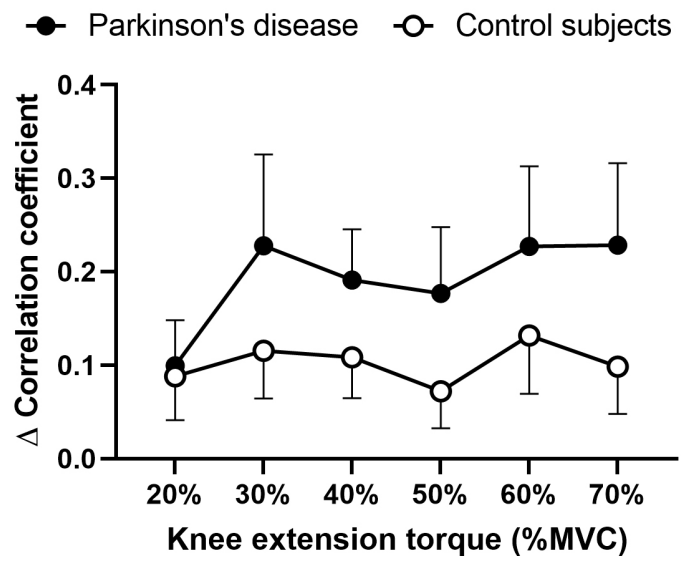

Fig. 6. Comparison of the $\Delta$ correlation coefficient between PD subjects and controls. PD subjects showed a significantly higher $\Delta$ correlation coefficient than control subjects during the submaximal ramp-up contraction task. Data are presented as mean $\pm 95 \%$ CI.

(4) the temporal changes in spatial MU activation properties showed significant laterality in people with PD compared with healthy controls. On the other hand, the control group did not show laterality in the muscle activation pattern. These results support the hypothesis that people with PD show laterality of MU activation properties when compared with control subjects during force production (low to high-intensity contraction).

These findings support the hypothesis that the moreaffected side of people with PD shows higher amplitude and lower heterogeneity than the contralateral side of people with PD and control subjects during the force production (low to high-intensity contraction).

The knee extension torque of the more-affected side of people with PD was significantly lower than that of the contralateral side in people with PD and control subjects in the present study. This finding is consistent with the findings of previous studies that showed asymmetries in lower limb muscle strength $[29,30]$. The muscle weakness and asymmetrical motor symptoms of people with $\mathrm{PD}$ may be related to the asymmetry of dopamine in the SNPc, resulting in extrapyramidal dysfunction and impaired motor output [31-33]. Consequently, the results of the present study may reflect asymmetry in neurodegeneration. Although people with PD who take oral medications that compensate for the reduced level of dopamine neurons exhibit improved motor function $[28,34]$, here, it is shown that asymmetric motor patterns persist.

The results of this study showed significantly higher relative RMS values on the more-affected side of people with PD than on the contralateral side of people with PD and control subjects. Furthermore, the more affected side showed moderate correlations between the scores of UPDRS part III and $\Delta$ normalized RMS and $\Delta \mathrm{CoV}$ of the RMS and $\Delta$ modified entropy. As shown in previous studies, the moreaffected side of people with PD and people with PD in the off- medication state showed higher RMS values than either side of age-matched healthy subjects and people with PD in the on-medication state, respectively $[6,28]$. A previous study reported that reticulospinal pathways originating in the nucleus reticularis gigantocellularis were disinhibited in people with PD, resulting in hyperexcitability of spinal interneurons [35]. Therefore, abnormal muscle tone, such as rigidity, occurs due to the degeneration of the reticulospinal pathways and the degeneration of the SNPc. The results of the present study suggest asymmetry in SNPc degeneration.

Less heterogeneity (lower CoV of the RMS and higher modified entropy) is reported for the more-affected side of people with $\mathrm{PD}$ than the contralateral side of people with PD and control subjects during the submaximal ramp-up contraction task. A higher level of heterogeneity in the EMG signal is consistent with a more coordinated and consistent activation of the lower limb muscle [21]. Based on this finding, the more-affected side of people with PD exhibited more inefficient muscle activity than the contralateral side of people with PD and control subjects. People with PD presenting without tremor show a lower amplitude of variance in mechanical muscle oscillations and acceleration than control subjects [36]. Motor symptoms occur after a marked loss of nigral neurons, resulting in the depletion of striatal dopamine, especially on the side of disease onset [37]. A previous study using positron emission tomography showed a correlation between biochemical asymmetry in the caudate and putamen and motor symptoms in people with early PD [38]. Furthermore, the activity of the putamen and supplementary motor area during unilateral movement is significantly reduced in these individuals and the authors suggested that the asymmetrical brain activity pattern may be an underlying reason for some motor deficits [39]. The central nervous system (e.g., nigral neurons and putamen) indirectly regulates force output by modulating the cortical recruitment of MUs and their firing rates [3]. At the level of the MU, a previous study reported that the more-affected side of people with PD exhibited more MU activity at lower force thresholds and inconsistent discharge rates of MUs than participants without PD in a submaximal task [4]. Dengler et al. [40] also reported that people with PD exhibit irregular firing patterns of MUs in the early stage of the disease. Thus, the loss of neurons in the SNPc not only increases muscle tone but also increases the irregular firing patterns of MUs. Furthermore, people with PD exhibited temporal changes in spatial MU activation properties and showed significant laterality when compared to healthy controls in both low and high-intensity contractions. In addition to degeneration of the central nervous system, people with PD display selective atrophy of fast-twitch muscle fibers [9]. This finding suggests that people with PD are impaired in the recruitment of MUs during high-intensity muscle contraction. In this study, there was clear muscle weakness on the more-affected side of the people with $\mathrm{PD}$, which supports the idea that their MU activation properties are impaired during high-intensity muscle 
contraction. Although aerobic exercise is often implemented as physical therapy for people with $\mathrm{PD}$, high-intensity muscle strength training is also expected to be useful, considering the specifics of the disability caused by PD.

This study has several limitations. First, only females were recruited. As shown in a previous study, compared with healthy young males, heathy females exhibited larger differences in the spatial distribution patterns of a sustained isometric contraction [22]. However, no studies have compared muscle activation patterns between females and males with PD. Second, only people with PD presenting mild motor symptoms were recruited for this study. Patients with different severities of PD must be compared to determine whether the laterality of EMG variables is affected by the disease stage or disease progression.

\section{Conclusions}

In this study, the laterality of spatial distributions of HDSEMG signal amplitudes between bilateral sides of people with PD and control subjects was compared during force production (low- to high-intensity contraction). Based on the results of this study, the more-affected side in people with PD exhibits a higher RMS value and lower heterogeneity than the contralateral side in people with PD or either side in controls. In particular, the temporal changes in spatial MU activation properties showed significant laterality in people with PD compared to healthy control subjects, not only for low-intensity contractions but also for high-intensity contractions. These findings suggest that people with PD have asymmetrical MU activation properties, independent of the magnitude of force production.

\section{Abbreviations}

$\mathrm{CoV}$, coefficient of variation; EMG, electromyography; HD-SEMG, High-density surface electromyography; MU, motor unit; MVIC, maximal voluntary isometric contraction; PD, Parkinson's disease; RMS, root mean square; SEMG, surface electromyography; SNPc, substantia nigra parts compacta; UPDRD, Unified Parkinson's disease rating scale; VL, vastus lateralis.

\section{Author contributions}

YN and KW conceived and designed the study; YN performed experiments; YN and KW analyzed data; YN, KW, TT, NM, HM, ST, and AH interpreted the results of experiments; YN, KW and AH prepared figures; YN, KW, TT, and AH drafted the manuscript; and YN, KW, TT, NM, HM, $\mathrm{ST}$, and $\mathrm{AH}$ edited and revised the manuscript. YN, KW, TT, NM, HM, ST, and AH approved the final version of the manuscript.

\section{Ethics approval and consent to participate}

All experimental procedures were performed in accordance with the Declaration of Helsinki. This research protocol was approved by the Hiroshima University Committee on Ethics in Research (approval number, E-53-2). All participants received verbal and written explanations about their participation in this study and publication of this article and signed a consent form.

\section{Acknowledgment}

We thank Naoya Orita and Masashi Shimada for technical assistance.

\section{Funding}

This study was supported in part by research grants from JSPS KAKENHI, Grants-in-Aid for Young Scientists (17K179080 and 20K19448).

\section{Conflict of interest}

The authors declare no conflict of interest.

\section{References}

[1] Kalia LV, Lang AE. Parkinson's disease. Lancet. 2015; 386: 896912.

[2] Stevens-Lapsley J, Kluger BM, Schenkman M. Quadriceps muscle weakness, activation deficits, and fatigue with Parkinson disease. Neurorehabilitation and Neural Repair. 2012; 26: 533-541.

[3] De Luca CJ, LeFever RS, McCue MP, Xenakis AP. Behaviour of human motor units in different muscles during linearly varying contractions. Journal of Physiology. 1982; 329: 113-128.

[4] Glendinning DS, Enoka RM. Motor unit behavior in Parkinson's disease. Physical Therapy. 1994; 74: 61-70.

[5] Nishikawa Y, Watanabe K, Holobar A, Maeda N, Maruyama H, Tanaka S. Identification of the laterality of motor unit behavior in female patients with parkinson's disease using high-density surface electromyography. European Journal of Neuroscience. 2021; 53: 1938-1949.

[6] Nishikawa Y, Watanabe K, Takahashi T, Orita N, Kimura H, Matsumoto $\mathrm{M}$, et al. Spatial electromyography distribution pattern of the vastus lateralis muscle during ramp up contractions in Parkinson's disease patients. Journal of Electromyography and Kinesiology. 2017; 37: 125-131.

[7] Henneman E, Somjen G, Carpenter DO. Functional significance of cell size in spinal motoneurons. Journal of Neurophysiology. 1965; 28: 560-580.

[8] Djaldetti R, Ziv I, Melamed E. The mystery of motor asymmetry in Parkinson's disease. Lancet Neurology. 2006; 5: 796-802.

[9] Edström L. Selective changes in the sizes of red and white muscle fibres in upper motor lesions and Parkinsonism. Journal of the Neurological Sciences. 1970; 11: 537-550.

[10] Holobar A, Farina D. Blind source identification from the multichannel surface electromyogram. Physiological Measurement. 2014; 35: R143-R165.

[11] Negro F, Muceli S, Castronovo AM, Holobar A, Farina D. Multichannel intramuscular and surface EMG decomposition by convolutive blind source separation. Journal of Neural Engineering. 2017; 13: 026027.

[12] Barbero M, Falla D, Mafodda L, Cescon C, Gatti R. The location of peak upper trapezius muscle activity during submaximal contractions is not associated with the location of myofascial trigger points: new insights revealed by high-density surface EMG. Clinical Journal of Pain. 2016; 32: 1044-1052.

[13] Gaffney BM, Maluf KS, Curran-Everett D, Davidson BS. Associations between cervical and scapular posture and the spatial distribution of trapezius muscle activity. Journal of Electromyography and Kinesiology. 2014; 24: 542-549.

[14] Castroflorio T, Falla D, Wang K, Svensson P, Farina D. Effect of experimental jaw-muscle pain on the spatial distribution of surface 
EMG activity of the human masseter muscle during tooth clenching. Journal of Oral Rehabilitation. 2012; 39: 81-92.

[15] Chanaud CM, Macpherson JM. Functionally complex muscles of the cat hindlimb. III. Differential activation within biceps femoris during postural perturbations. Experimental Brain Research. 1991; 85: 271-280.

[16] Lexell J, Downham DY. The occurrence of fibre-type grouping in healthy human muscle: a quantitative study of cross-sections of whole vastus lateralis from men between 15 and 83 years. Acta Neuropathologica. 1991; 81: 377-381.

[17] Holtermann A, Roeleveld K, Karlsson JS. Inhomogeneities in muscle activation reveal motor unit recruitment. Journal of Electromyography and Kinesiology. 2005; 15: 131-137.

[18] Holtermann A, Roeleveld K, Mork PJ, Grönlund C, Karlsson JS, Andersen LL, et al. Selective activation of neuromuscular compartments within the human trapezius muscle. Journal of Electromyography and Kinesiology. 2009; 19: 896-902.

[19] Szewczyk-Krolikowski K, Tomlinson P, Nithi K, Wade-Martins $\mathrm{R}, \mathrm{Talbot} \mathrm{K}$, Ben-Shlomo $\mathrm{Y}$, et al. The influence of age and gender on motor and non-motor features of early Parkinson's disease: initial findings from the Oxford Parkinson Disease Center (OPDC) discovery cohort. Parkinsonism \& Related Disorders. 2014; 20: 99-105.

[20] Nishikawa Y, Watanabe K, Kawade S, Takahashi T, Kimura H, Maruyama $\mathrm{H}$, et al. The effect of a portable electrical muscle stimulation device at home on muscle strength and activation patterns in locomotive syndrome patients: a randomized control trial. Journal of Electromyography and Kinesiology. 2019; 45: 46-52.

[21] Watanabe K, Kouzaki M, Merletti R, Fujibayashi M, Moritani T. Spatial EMG potential distribution pattern of vastus lateralis muscle during isometric knee extension in young and elderly men. Journal of Electromyography and Kinesiology. 2012; 22: 74-79.

[22] Nishikawa Y, Watanabe K, Takahashi T, Hosomi N, Orita N, Mikami $\mathrm{Y}$, et al. Sex differences in variances of multi-channel surface electromyography distribution of the vastus lateralis muscle during isometric knee extension in young adults. European Journal of Applied Physiology. 2017; 117: 583-589.

[23] Watanabe K, Miyamoto T, Tanaka Y, Fukuda K, Moritani T. Type 2 diabetes mellitus patients manifest characteristic spatial EMG potential distribution pattern during sustained isometric contraction. Diabetes Research and Clinical Practice. 2012; 97: 468-473.

[24] Farina D, Leclerc F, Arendt-Nielsen L, Buttelli O, Madeleine P. The change in spatial distribution of upper trapezius muscle activity is correlated to contraction duration. Journal of Electromyography and Kinesiology. 2008; 18: 16-25.

[25] Holtermann A, Grönlund C, Stefan Karlsson J, Roeleveld K. Spatial distribution of active muscle fibre characteristics in the upper trapezius muscle and its dependency on contraction level and duration. Journal of Electromyography and Kinesiology. 2008; 18: 372-381.

[26] Watanabe K, Kouzaki M, Moritani T. Spatial EMG potential dis- tribution of biceps brachii muscle during resistance training and detraining. European Journal of Applied Physiology. 2015; 115: 2661-2670.

[27] Hyngstrom AS, Murphy SA, Nguyen J, Schmit BD, Negro F, Gutterman DD, et al. Ischemic conditioning increases strength and volitional activation of paretic muscle in chronic stroke: a pilot study. Journal of Applied Physiology. 2018; 124: 1140-1147.

[28] Nishikawa Y, Watanabe K, Takahashi T, Kimura H, Maruyama H. The effect of medication on vastus lateralis muscle activation patterns in Parkinson's disease patients. Journal of Electromyography and Kinesiology. 2018; 42: 66-73.

[29] Nogaki H, Kakinuma S, Morimatsu M. Movement velocity dependent muscle strength in Parkinson's disease. Acta Neurologica Scandinavica. 1999; 99: 152-157.

[30] Nogaki H, Kakinuma S, Morimatsu M. Muscle weakness in Parkinson's disease: a follow-up study. Parkinsonism \& Related Disorders. 2001; 8: 57-62.

[31] Koller W, Kase S. Muscle strength testing in Parkinson's disease. European Neurology. 1986; 25: 130-133.

[32] Kempster PA, Gibb WR, Stern GM, Lees AJ. Asymmetry of substantia nigra neuronal loss in Parkinson's disease and its relevance to the mechanism of levodopa related motor fluctuations. Journal of Neurology, Neurosurgery, and Psychiatry. 1989; 52: 72-76.

[33] Tissingh G, Booij J, Bergmans P, Winogrodzka A, Janssen AG, van Royen EA, et al. Iodine-123-N-omega-fluoropropyl-2betacarbomethoxy-3beta-(4-iod ophenyl)tropane SPECT in healthy controls and early-stage, drug-naive Parkinson's disease. Journal of Nuclear Medicine. 1998; 39: 1143-1148.

[34] Brown P, Corcos DM, Rothwell JC. Does parkinsonian action tremor contribute to muscle weakness in Parkinson's disease? Brain. 1997; 120: 401-408.

[35] Chronister RB, Walding JS, Aldes LD, Marco LA. Interconnections between substantia nigra reticulata and medullary reticular formation. Brain Research Bulletin. 1988; 21: 313-317.

[36] Schaefer LV, Bittmann FN. Parkinson patients without tremor show changed patterns of mechanical muscle oscillations during a specific bilateral motor task compared to controls. Scientific Reports. 2020; 10: 1168.

[37] Riederer P, Jellinger KA, Kolber P, Hipp G, Sian-Hülsmann J, Krüger R. Lateralisation in Parkinson disease. Cell and Tissue Research. 2018; 373: 297-312.

[38] Kumakura Y, Gjedde A, Danielsen EH, Christensen S, Cumming P. Dopamine storage capacity in caudate and putamen of patients with early Parkinson's disease: correlation with asymmetry of motor symptoms. Journal of Cerebral Blood Flow and Metabolism. 2006; 26: 358-370.

[39] Wu T, Hou Y, Hallett M, Zhang J, Chan P. Lateralization of brain activity pattern during unilateral movement in Parkinson's disease. Human Brain Mapping. 2015; 36: 1878-1891.

[40] Dengler R, Wolf W, Schubert M, Struppler A. Discharge pattern of single motor units in basal ganglia disorders. Neurology. 1986; 36: 1061-1066. 